\title{
Introduction to the special issue: information and communications technology for crisis management: defining an agenda for scientific research
}

\author{
David Mendonça $\cdot$ Harry Bouwman
}

Published online: 11 March 2011

(C) Springer-Verlag London Limited 2011

Information and communication technology (ICT) for postdisaster management of critical infrastructure functions best when its design follows from a solid understanding of the sociotechnical systems that it is meant to support. Yet as is evident from past (Quarantelli 1997) and more current research (National Research Council 2007), there are significant gaps in scientific understanding concerning how ICT should be designed (Computer Science and Telecommunications Board 1996), used (Stewart and Bostrom 2002), and evaluated (Orlikowski and Iacono 2001). ICT often provides the means by which data, information, and knowledge are shared and used across critical infrastructure systems. Contributions to ICT research in crisis management therefore bear directly upon questions of how to analyze, design, and manage critical infrastructure systems.

Developing an agenda for fundamental ICT-centered research in the domain of crisis management is likely to require input from multiple disciplines. The papers in this

This work was completed while David Mendonça was a visiting professor at Delft University of Technology.

D. Mendonça $(\bowtie)$

Industrial and Systems Engineering Department,

Rensselaer Polytechnic Institute, Troy, NY 12180, USA

e-mail: mendod@rpi.edu

H. Bouwman

Information and Communication Technology Section,

Delft University of Technology, 2600 GA Delft,

The Netherlands

e-mail: w.a.g.a.bouwman@tudelft.nl

H. Bouwman

Institute for Advanced Management Systems Research,

Åbo Akademi, Turku, Finland special issue are the result of a convergence of workshopbased discussions among researchers. These discussions, and the various working papers that led to the contributed articles, centered on three questions (Stewart and Bostrom 2002):

- What do we know? What do we know about the role of ICT in crisis management? For example, what circumstances can influence success or failure in design, development, and use?

- What do we need to know? Identify questions that are likely to be salient over the next 10-30 years. For example, approaches to instrumenting the environment for extreme event prediction/management, design and evaluation of technologies to support communication, decision making and learning by involved persons and organizations.

- How are we going to find the answers? Preliminary discussion of possible funding sources, new research methods, approaches to develop strategic partnerships, and/or create shared data sets.

A clear point product of the authors' work in addressing these questions is an articulated need for improved measurement of key constructs in research on ICT and crisis management. To assess the impact of ICT on crisis, a robust set of measures is required that can be applied over time and space, and with respect to many and divers event types.

There are examples from other areas where reliable and valid measures of key constructs have helped to sustain research in these areas (e.g., assessments of economic vulnerability). The development of such measures in the area of crisis management would enable benchmarking (e.g., of efficacy of planning, response, and recovery efforts) and enhance credibility of work in the 
domain for policy makers and the general public. Three different constructs received considerable attention during the workshop: control, trust, and system state. Control is a central problem in crisis management. A number of challenges were raised to control in crisis management, such as what are appropriate measures for determining whether planning, response, and recovery processes are (i) operating within tolerance limits and (ii) not subject to non-random sources of error?

A second recurring construct in need of operationalization was trust. The question of how to measure trust arose in discussions of single operators using technologies, through policy makers attempting to assess the information provided to them from the field. In the first case, given the complexity of much ICT, at which points of contact between human and technology do (and should) trust occur? A driving question was what do users need to be able to see or explore in order to trust the technology?

The third important construct in need of measurement was system state. Participants recognized the need for viewing crisis management as a multidisciplinary endeavor, and the task of doing research on ICT in crisis management as therefore requiring a similarly multidisciplinary approach. A number of opportunities for future research were identified in measuring the state of sociotechnical systems (e.g., critical infrastructures). Specific examples included benchmarking of performance (e.g., reliability) of critical infrastructure systems, though other less obvious examples (such as management trust in such systems) were also discussed.

\section{Validation}

There is a great deal of skepticism about much ICTrelated research in the domain of crisis management concerning the external validity of claimed results (that is, the extent to which the results of a study apply beyond the bounds of the study design). Endemic problems were use of inappropriate test scenarios (e.g., use of toy problems), experiment participants (naive instead of experienced personnel), and periods of study (an afternoon versus multiple years). Countervailing examples are available, but these are far from being either established as golden standards or being known in the ICT area. On the other hand, there are very real challenges involved in assessing the external validity of results for the use of tools in managing high-impact, low-probability events. From a more positive perspective, research specifically designed to test the validity of some key theories in the crisis management is evolving.

\section{Theory development}

There is a fundamental difference in research philosophy between theory-driven and problem-driven research. On the one hand, theory-driven approaches by definition engage existing bodies of knowledge and may strongly imply choices about methodological approach. On the other hand, they may limit the scope of inquiry to familiar territory, thus hindering scientific progress. Similarly, problem-driven approaches assume that new questions that are not covered by current theories are constantly being revealed; however, it is only with careful analysis of prior research that claims of problem originality or newness can be addressed.

Despite the considerable experience of researchers working with practitioners, the point is often made that the research community needs to deepen its engagement with practitioners and indeed to put more weight on contributions of research to practice. To make this work will require honest assessments of how collaborations between research and practice can be mutually beneficial (a number of positive as well as negative examples were cited). Training sessions, such as the large-scale exercises that have been held in recent years, offer one opportunity for researchers to demonstrate value added, while also contributing to the scientific basis of the training itself.

\section{Finding out the answers}

The following mechanisms for encouraging or enabling multidisciplinary work in this area are relevant:

\subsection{Funding}

The research being proposed might not fit neatly within disciplinary categories. Special programs (such as the National Science Foundation's recent program in Human and Systems Dynamics) may need to be created.

\subsection{Research community}

A research community-with high quality journals, conferences, and network of departments-is needed to sustain work in this area. At present, the Information Systems for Crisis Response and Management community helps fulfill the second of these needs.

\subsection{High-impact research programs}

Research that contributes directly to practice in measurable ways is sorely needed. 


\subsection{Test beds}

There are few opportunities for multidisciplinary research teams to observe (and perhaps intervene) in real- or simulated-world settings related to crisis management. Without these test beds, research will be limited in scale.

\subsection{Education \& training programs}

A sometimes overlooked component of the development of research in ICT for crisis management is that of education, particularly at the undergraduate, graduate, and professional levels. Conducting multidisciplinary research and practice requires skill sets that are not found in common across a sufficient breadth of individual disciplines (e.g., simulation in social science curricula and survey-based methods in engineering curricula).

The papers presented in this issue address a number of these questions and issues, while articulating suggestions for future research. All the papers offer concrete examples from current and prior ICT systems to illustrate concepts.

Walker et al. discuss possible extensions of prior research on the use of gaming simulations for work on crisis management, focusing in particular on how these simulations can support training and learning. The paper provides a valuable historical perspective, encompassing early work in the field, and clearly tracing trends and challenges through to the present.

In a far-ranging review paper, Schraagen et al. place human factors considerations squarely in the center of ICTfocused research in crisis management. They identify new challenges for work in this area-particularly in the construction and validation of tools to support the development of situation awareness and critical thinking.

Nemeth et al. offer a detailed case study of ICT-based support for achieving resilient operations in critical care situations. Their approach enables low-level cognitive and behavioral processes to be revealed, clearly delineating the conceptual and practical links between study instrumentation and theoretical constructs for work in this area.

Bharosa et al. offer a far-ranging exploration of the link between information quality and organizational performance during crisis response, as mediated by ICT. The result is an ICT architecture that is closely tied to analysis of crisis response tasks and archetypical organizational structures.

Taken together, the papers included in this special issue squarely confront questions of design, validation, and evaluation of ICT systems for crisis management. A particular strength of this work is its engagement and synthesis of a large and diffuse literature. The papers also offer working models of how multidisciplinary perspectives may be brought to bear in addressing perennial issues in this area. Finally, we hope that this work will prove useful to researchers in identifying promising directions for future research.

Acknowledgments This material is based upon work supported by Delft University of Technology and by the U.S. National Science Foundation under Grant No. CMS-0449582.

\section{References}

Computer Science and Telecommunications Board (1996) Computing and telecommunications in the extreme. National Research Council, Washington

National Research Council (2007) Improving disaster management: the role of it in mitigation, preparedness, response and recovery. The National Academies Press, Washington

Orlikowski W, Iacono CS (2001) Desperately seeking the "IT" in IT research: a call to theorizing the IT artifact. Inform Sys Res 12:121-134

Quarantelli EL (1997) Problematical aspects of the information/ communication revolution for disaster planning and research: ten non-technical issues and questions. Disaster Prev Manag 6:94-106

Stewart TR, Bostrom A (2002) Extreme event decision making: workshop report. University at Albany, Albany 